\title{
Erratum to: Increased risk of chronic fatigue syndrome following herpes zoster: a population-based study
}

\author{
S.-Y. Tsai • T.-Y. Yang • H.-J. Chen • C.-S. Chen • \\ W.-M. Lin • W.-C. Shen • C.-N. Kuo • C.-H. Kao
}

Accepted: 17 April 2014 / Published online: 1 May 2014

(C) Springer-Verlag Berlin Heidelberg 2014

\section{Erratum to: Eur J Clin Microbiol Infect Dis \\ DOI 10.1007/s10096-014-2095-x}

In three sentences, two in the second paragraph of the Discussion and one in the final paragraph of the Discussion, the acronym CFS was falsely rendered as CSF. The authors and the publisher apologize for these errors.

The online version of the original article can be found at http://dx.doi.org/ 10.1007/s10096-014-2095-x.

S.-Y. Tsai

Department of Laboratory Medicine, Mackay Memorial Hospital,

Taipei, Taiwan

\section{S.-Y. Tsai}

Department of Health Policy and Management, Bloomberg School

of Public Health, Johns Hopkins University, Baltimore, MD, USA

T.-Y. Yang

Molecular and Genomic Epidemiology Center, China Medical

University Hospital, China Medical University, Taichung, Taiwan

\section{H.-J. Chen}

Management Office for Health Data, China Medical University

Hospital, Taichung, Taiwan

H.-J. Chen

Department of Public Health, China Medical University, Taichung, Taiwan

C.-S. Chen

Division of Chinese Trauma, China Medical University Hospital, China Medical University, Taichung, Taiwan
W.-M. Lin

Department of Diagnostic Radiology, Chang Gung Memorial Hospital, Chiayi, Taiwan

W.-M. Lin

Chang Gung University, Tao Yuan, Taiwan

W.-C. Shen

Department of Computer Science and Information Engineering, Asia University, Taichung, Taiwan

C.-N. Kuo

Kau-Tang Traditional Medical Hospital, Taichung, Taiwan

C.-H. Kao

Department of Nuclear Medicine and PET Center, China Medical

University Hospital, Taichung, Taiwan

C.-H. Kao $(\square)$

Graduate Institute of Clinical Medicine Science and School of Medicine, College of Medicine, China Medical University, No. 2,

Yuh-Der Road, Taichung 404, Taiwan

e-mail: d10040@mail.cmuh.org.tw 